\title{
Positive solutions for a coupled system of nonlinear differential equations of mixed fractional orders
}

Yige Zhao ${ }^{1}$, Shurong Sun ${ }^{1,2^{*}}$, Zhenlai Han ${ }^{1,3}$ and Wenquan Feng ${ }^{1}$

\author{
* Correspondence: sshrong@163. \\ com \\ ${ }^{1}$ School of Science, University of \\ Jinan, Jinan 250022, Shandong, PR \\ China \\ Full list of author information is \\ available at the end of the article
}

\begin{abstract}
In this article, we study the existence of positive solutions for a coupled system of nonlinear differential equations of mixed fractional orders

$$
\left\{\begin{array}{l}
-D_{0^{+}}^{\alpha} u(t)=f(t, v(t)), 0<t<1, \\
D_{0^{+}}^{\beta} v(t)=g(t, u(t)), \quad 0<t<1, \\
u(0)=u(1)=u^{\prime}(0)=v(0)=v(1)=v^{\prime}(0)=v^{\prime}(1)=0,
\end{array}\right.
$$

where $2<\alpha \leq 3,3<\beta \leq 4, D_{0^{+}}^{\alpha}, D_{0^{+}}^{\beta}$ are the standard Riemann-Liouville fractional derivative, and $f, g:[0,1] \times[0,+\infty) \rightarrow[0,+\infty)$ are given continuous functions, $f(t, 0)$ $\equiv 0, g(t, 0) \equiv 0$. Our analysis relies on fixed point theorems on cones. Some sufficient conditions for the existence of at least one or two positive solutions for the boundary value problem are established. As an application, examples are presented to illustrate the main results.
\end{abstract}

Keywords: Positive solution, coupled system, fractional Green?'?s function, fixed point theorem

\section{Introduction}

Fractional differential equations have been of great interest recently. It is caused by the both intensive development of the theory of fractional calculus itself and applications, see [1-6]. Recently, there are a large number of papers dealing with the existence of solutions of nonlinear fractional differential equations by the use of techniques of nonlinear analysis (fixed point theorems, Leray-Schauder theory, Adomian decomposition method, etc.), see [7-21]. The articles [13-21] considered boundary value problems for fractional differential equations.

$\mathrm{Yu}$ and Jiang [20] examined the existence of positive solutions for the following problem

$$
\begin{gathered}
D_{0^{+}}^{\alpha} u(t)+f(t, u(t))=0,0<t<1, \\
u(0)=u(1)=u^{\prime}(0)=0,
\end{gathered}
$$

where $2<\alpha \leq 3$ is a real number, $f \in C([0,1] \times[0,+\infty) ;(0,+\infty))$ and $D_{0^{+}}^{\alpha}$ is the Riemann-Liouville fractional differentiation. Using the properties of the Green function, they obtained some existence criteria for one or two positive solutions for

\section{SpringerOpen $^{\circ}$}

(c) 2011 Zhao et al; licensee Springer. This is an Open Access article distributed under the terms of the Creative Commons Attribution License (http://creativecommons.org/licenses/by/2.0), which permits unrestricted use, distribution, and reproduction in any medium, provided the original work is properly cited. 
singular and nonsingular boundary value problems by means of the Krasnosel'skii fixed point theorem and a mixed monotone method.

$\mathrm{Xu}$ et al. [21] considered the existence of positive solutions for the following problem

$$
\begin{gathered}
D_{0^{+}}^{\alpha} u(t)=f(t, u(t)), 0<t<1, \\
u(0)=u(1)=u^{\prime}(0)=u^{\prime}(1)=0,
\end{gathered}
$$

where $3<\alpha \leq 4$ is a real number, $f \in C([0,1] \times[0,+\infty),(0,+\infty))$ and $D_{0^{+}}^{\alpha}$ is the Riemann-Liouville fractional differentiation. Using the properties of the Green function, they gave some multiple positive solutions for singular and nonsingular boundary value problems, and also they gave uniqueness of solution for singular problem by means of Leray-Schauder nonlinear alternative, a fixed point theorem on cones and a mixed monotone method.

On the other hand, the study of coupled systems involving fractional differential equations is also important as such systems occur in various problems, see [22-30].

Bai and Fang [24] considered the existence of positive solutions of singular coupled system

$$
\left\{\begin{array}{l}
D^{s} u=f(t, v), 0<t<1, \\
D^{p} v=g(t, u), 0<t<1,
\end{array}\right.
$$

where $0<s, p<1$, and $f, g:[0,1) \times[0,+\infty) \rightarrow[0,+\infty)$ are two given continuous functions, $\lim _{t \rightarrow 0^{+}} f(t, \cdot)=+\infty, \lim _{t \rightarrow 0^{+}} g(t, \cdot)=+\infty$ and $D^{s}, D^{p}$ are two standard Riemann-Liouville fractional derivatives. They established the existence results by a nonlinear alternative of Leray-Schauder type and Krasnosel'skii fixed point theorem on a cone.

$\mathrm{Su}$ [25] discussed a boundary value problem for a coupled differential system of fractional order

$$
\left\{\begin{array}{l}
D^{\alpha} u(t)=f\left(t, v(t), D^{\mu} v(t)\right), 0<t<1 \\
D^{\beta} v(t)=g\left(t, u(t), D^{v} u(t)\right), 0<t<1 \\
u(0)=u(1)=v(0)=v(1)=0
\end{array}\right.
$$

where $1<\alpha, \beta \leq 2, \mu, v>0, \alpha-v \geq 1, \beta-\mu \geq 1, f, g:[0,1] \times \mathbb{R} \times \mathbb{R} \rightarrow \mathbb{R}$ are given functions and $D$ is the standard Riemann-Liouville fractional derivative. By means of Schauder fixed point theorem, an existence result for the solution was obtained.

From the above works, we can see a fact, although the coupled systems of fractional boundary value problems have been investigated by some authors, coupled systems due to mixed fractional orders are seldom considered. Motivated by all the works above, in this article we investigate the existence of positive solutions for a coupled system of nonlinear differential equations of mixed fractional orders

$$
\left\{\begin{array}{l}
-D_{0^{+}}^{\alpha} u(t)=f(t, v(t)), 0<t<1, \\
D_{0^{+}}^{\beta} v(t)=g(t, u(t)), \quad 0<t<1, \\
u(0)=u(1)=u^{\prime}(0)=v(0)=v(1)=v^{\prime}(0)=v^{\prime}(1)=0,
\end{array}\right.
$$

where $2<\alpha \leq 3,3<\beta \leq 4, D_{0^{+}}^{\alpha}, D_{0^{+}}^{\beta}$ are the standard Riemann-Liouville fractional derivative, and $f, g:[0,1] \times[0,+\infty) \rightarrow[0,+\infty)$ are given continuous functions, $f(t, 0) \equiv$ $0, g(t, 0) \equiv 0$. Our analysis relies on fixed point theorems on cones. Some sufficient conditions for the existence of at least one or two positive solutions for the boundary 
value problem are established. Finally, we present some examples to demonstrate our results.

The article is organized as follows. In Sect. 2, we shall give some definitions and lemmas to prove our main results. In Sect. 3, we establish existence results of at least one or two positive solutions for boundary value problem (1.1) by fixed point theorems on cones. In Sect. 4, examples are presented to illustrate the main results.

\section{Preliminaries}

For the convenience of readers, we give some background materials from fractional calculus theory to facilitate analysis of problem (1.1). These materials can be found in the recent literature, see [20,21,31-33].

Definition 2.1 [31] The Riemann-Liouville fractional derivative of order $\alpha>0$ of a continuous function $f:(0,+\infty) \rightarrow \mathbb{R}$ is given by

$$
D_{0^{+}}^{\alpha} f(t)=\frac{1}{\Gamma(n-\alpha)}\left(\frac{\mathrm{d}}{\mathrm{d} t}\right)^{(n)} \int_{0}^{t} \frac{f(s)}{(t-s)^{\alpha-n+1}} \mathrm{~d} s,
$$

where $n=[\alpha]+1,[\alpha]$ denotes the integer part of number $\alpha$, provided that the right side is pointwise defined on $(0,+\infty)$.

Definition 2.2 [31] The Riemann-Liouville fractional integral of order $\alpha>0$ of a function $f:(0,+\infty) \rightarrow \mathbb{R}$ is given by

$$
I_{0^{+}}^{\alpha} f(t)=\frac{1}{\Gamma(\alpha)} \int_{0}^{t}(t-s)^{\alpha-1} f(s) \mathrm{d} s,
$$

provided that the right side is pointwise defined on $(0,+\infty)$.

From the definition of the Riemann-Liouville derivative, we can obtain the following statement.

Lemma 2.1 [31] Let $\alpha>0$. If we assume $u \in C(0,1) \cap L(0,1)$, then the fractional differential equation

$$
D_{0^{+}}^{\alpha} u(t)=0
$$

has $u(t)=c_{1} t^{\alpha-1}+c_{2} t^{\alpha-2}+\ldots+c_{n} t^{\alpha-n}, c_{i} \in \mathbb{R}, i=1,2, \ldots, n$, as unique solutions, where $n$ is the smallest integer greater than or equal to $\alpha$.

Lemma 2.2 [31] Assume that $u \in C(0,1) \cap L(0,1)$ with a fractional derivative of order $\alpha>0$ that belongs to $C(0,1) \cap L(0,1)$. Then

$$
I_{0^{+}}^{\alpha} D_{0^{+}}^{\alpha} u(t)=u(t)+c_{1} t^{\alpha-1}+c_{2} t^{\alpha-2}+\cdots+c_{n} t^{\alpha-n}, \text { for some } c_{i} \in \mathbb{R}, \quad i=1,2, \ldots, n,
$$

where $n$ is the smallest integer greater than or equal to $\alpha$.

In the following, we present the Green function of fractional differential equation boundary value problem.

Lemma $2.3[20]$ Let $h_{1} \in C[0,1]$ and $2<\alpha \leq 3$. The unique solution of problem

$$
\begin{aligned}
& -D_{0^{+}}^{\alpha} u(t)=h_{1}(t), \quad 0<t<1, \\
& u(0)=u(1)=u^{\prime}(0)=0,
\end{aligned}
$$




$$
u(t)=\int_{0}^{1} G_{1}(t, s) h_{1}(s) d s
$$

where

$$
G_{1}(t, s)= \begin{cases}\frac{t^{\alpha-1}(1-s)^{\alpha-1}-(t-s)^{\alpha-1}}{\Gamma(\alpha)}, & 0 \leq s \leq t \leq 1 \\ \frac{t^{\alpha-1}(1-s)^{\alpha-1}}{\Gamma(\alpha)}, & 0 \leq t \leq s \leq 1\end{cases}
$$

Here $G_{1}(t, s)$ is called the Green function of boundary value problem (2.1) and (2.2).

Lemma 2.4 [20] The function $G_{1}(t, s)$ defined by (2.3) satisfies the following conditions: (A1) $G_{1}(t, s)=G_{1}(1-s, 1-t)$, for $t, s \in(0,1)$;

(A2) $t^{\alpha-1}(1-t) s(1-s)^{\alpha-1} \leq \Gamma(\alpha) G_{1}(t, s) \leq(\alpha-1) s(1-s)^{\alpha-1}$, for $t, s \in(0,1)$;

(A3) $G_{1}(t, s)>0$, for $t, s \in(0,1)$;

(A4) $t^{\alpha-1}(1-t) s(1-s)^{\alpha-1} \leq \Gamma(\alpha) G_{1}(t, s) \leq(\alpha-1)(1-t) t^{\alpha-1}$, for $t, s \in(0,1)$.

Remark 2.1 Let $q_{1}(t)=t^{\alpha-1}(1-t), k_{1}(s)=s(1-s)^{\alpha-1}$. Then

$$
q_{1}(t) k_{1}(s) \leq \Gamma(\alpha) G_{1}(t, s) \leq(\alpha-1) k_{1}(s)
$$

Lemma $2.5[21]$ Let $h_{2} \in C[0,1]$ and $3<\beta \leq 4$. The unique solution of problem

$$
\begin{aligned}
& D_{0^{+}}^{\beta} u(t)=h_{2}(t), \quad 0<t<1 \\
& u(0)=u(1)=u^{\prime}(0)=u^{\prime}(1)=0,
\end{aligned}
$$

is

$$
u(t)=\int_{0}^{1} G_{2}(t, s) h_{2}(s) d s
$$

where

$$
G_{2}(t, s)= \begin{cases}\frac{(t-s)^{\beta-1}+(1-s)^{\beta-2} t^{\beta-2}[(s-t)+(\beta-2)(1-t) s]}{\Gamma(\beta)}, & 0 \leq s \leq t \leq 1, \\ \frac{t^{\beta-2}(1-s)^{\beta-2}[(s-t)+(\beta-2)(1-t) s]}{\Gamma(\beta)}, & 0 \leq t \leq s \leq 1 .\end{cases}
$$

Here $G_{2}(t, s)$ is called the Green function of boundary value problem (2.4) and (2.5).

Lemma 2.6 [21] The function $G_{2}(t, s)$ defined by (2.6) satisfies the following conditions:

(B1) $G_{2}(t, s)=G_{2}(1-s, 1-t)$, for $t, s \in(0,1)$;

(B2) $(\beta-2) t^{\beta-2}(1-t)^{2} s^{2}(1-s)^{\beta-2} \leq \Gamma(\beta) G_{2}(t, s) \leq M_{0} s^{2}(1-s)^{\beta-2}$, for $t, s \in(0,1)$;

(B3) $G_{2}(t, s)>0$, for $t, s \in(0,1)$;

(B4) $(\beta-2) s^{2}(1-s)^{\beta-2} t^{\beta-2}(1-t)^{2} \leq \Gamma(\beta) G_{2}(t, s) \leq M_{0} t^{\beta-2}(1-t)^{2}$, for $t, s \in(0,1)$, here $M_{0}=\max \left\{\beta-1,(\beta-2)^{2}\right\}$.

Remark 2.2 Let $q_{2}(t)=t^{\beta-2}(1-t)^{2}, k_{2}(s)=s^{2}(1-s)^{\beta-2}$. Then

$$
(\beta-2) q_{2}(t) k_{2}(s) \leq \Gamma(\beta) G_{2}(t, s) \leq M_{0} k_{2}(s) .
$$

The following two lemmas are fundamental in the proofs of our main results. 
Lemma 2.7 [32] Let $E$ be a Banach space, and let $P \subset E$ be a cone in E. Assume $\Omega_{1}$, $\Omega_{2}$ are open subsets of $E$ with $0 \in \Omega_{1} \subset \bar{\Omega}_{1} \subset \Omega_{2}$, and let $S: P \rightarrow P$ be a completely continuous operator such that, either

(D1) $\|S w\| \leq\|w\|, w \in P \cap \partial \Omega_{1},\|S w\| \geq\|w\|, w \in P \cap \partial \Omega_{2}$, or

(D2) $\|S w\| \geq\|w\|, w \in P \cap \partial \Omega_{1},\|S w\| \leq\|w\|, w \in P \cap \partial \Omega_{2}$.

Then $S$ has a fixed point in $P \cap\left(\bar{\Omega}_{2} \backslash \Omega_{1}\right)$.

Lemma 2.8 [33] Let $E$ be a Banach space, and let $P \subset E$ be a cone in E. Assume $\Omega_{1}$, $\Omega_{2}$ and $\Omega_{3}$ are open subsets of $E$ with $0 \in \Omega_{1} \subset \bar{\Omega}_{1} \subset \Omega_{2} \subset \bar{\Omega}_{2} \subset \Omega_{3}$, and let $S: P \cap\left(\bar{\Omega}_{3} \backslash \Omega_{1}\right) \rightarrow P$ be a completely continuous operator such that

(E1) $\|S w\| \geq\|w\|, \forall w \in P \cap \partial \Omega_{1}$;

(E2) $\|S w\| \leq\|w\|, S w \neq w, \forall w \in P \cap \partial \Omega_{2}$;

(E3) $\|S w\| \geq\|w\|, \forall w \in P \cap \partial \Omega_{3}$.

Then $S$ has two fixed points $w_{1}$ and $w_{2}$ in $P \cap\left(\bar{\Omega}_{3} \backslash \Omega_{1}\right)$ with $w_{1} \in\left(\bar{\Omega}_{2} \backslash \Omega_{1}\right)$ and $w_{2} \in\left(\bar{\Omega}_{3} \backslash \Omega_{2}\right)$.

\section{Main results}

In this section, we establish the existence results of positive solutions for boundary value problem (1.1).

Consider the following coupled system of integral equations:

$$
\left\{\begin{array}{l}
u(t)=\int_{0}^{1} G_{1}(t, s) f(s, v(s)) \mathrm{d} s, \\
v(t)=\int_{0}^{1} G_{2}(t, s) g(s, u(s)) \mathrm{d} s .
\end{array}\right.
$$

Lemma 3.1 Suppose that $f, g:[0,1] \times[0,+\infty) \rightarrow[0,+\infty)$ are continuous. Then $(u, v)$ $\in C[0,1] \times C[0,1]$ is a solution of $(1.1)$ if and only if $(u, v) \in C[0,1] \times C[0,1]$ is a solution of system (3.1).

This proof is similar to that of Lemma 3.3 in [25], so is omitted.

From (3.1), we can get the following integral equation

$$
u(t)=\int_{0}^{1} G_{1}(t, s) f\left(s, \int_{0}^{1} G_{2}(s, r) g(r, u(r)) \mathrm{d} r\right) \mathrm{d} s, \quad t \in[0,1] .
$$

Let Banach space $E=C[0,1]$ be endowed with the norm $\|u\|=\max _{0 \leq t \leq 1}|u(t)|$. De ne the cone $P \subset E$ by

$$
P=\left\{u \in E: u(t) \geq \frac{q_{1}(t)}{\alpha-1}\|u\|, \quad t \in[0,1]\right\} .
$$

We define an operator $T: P \rightarrow E$ as follows

$$
T u(t)=\int_{0}^{1} G_{1}(t, s) f\left(s, \int_{0}^{1} G_{2}(s, r) g(r, u(r)) \mathrm{d} r\right) \mathrm{d} s, \quad t \in[0,1] .
$$

Lemma 3.2 $T: P \rightarrow P$ is completely continuous. 
Proof. For $u \in P, 0 \leq t \leq 1$, by Lemma 2.4,

$$
\begin{aligned}
\|T u\| & =\max _{0 \leq t \leq 1}|T u(t)| \\
& =\max _{0 \leq t \leq 1}\left|\int_{0}^{1} G_{1}(t, s) f\left(s, \int_{0}^{1} G_{2}(s, r) g(r, u(r)) \mathrm{d} r\right) \mathrm{d} s\right| \\
& \leq \frac{\alpha-1}{\Gamma(\alpha)} \int_{0}^{1} k_{1}(s) f\left(s, \int_{0}^{1} G_{2}(s, r) g(r, u(r)) \mathrm{d} r\right) \mathrm{d} s, \\
T u(t) & =\int_{0}^{1} G_{1}(t, s) f\left(s, \int_{0}^{1} G_{2}(s, r) g(r, u(r)) \mathrm{d} r\right) \mathrm{d} s \\
& \geq \int_{0}^{1} \frac{q_{1}(t) k_{1}(s)}{\Gamma(\alpha)} f\left(s, \int_{0}^{1} G_{2}(s, r) g(r, u(r)) \mathrm{d} r\right) \mathrm{d} s \\
& \geq \frac{q_{1}(t)}{\alpha-1}\|T u\| .
\end{aligned}
$$

Thus we have $T(P) \subset P$.

The operator $T: P \rightarrow P$ is continuous in view of continuity of $G(t, s), f(t, u)$, and $g(t$, $u)$. For any bounded set $M, T(M)$ is uniformly bounded and equicontinuous. This proof is similar to that of Lemma 2.1.1 in [20], so is omitted. By means of ArzelaAscoli Theorem, $T: P \rightarrow P$ is completely continuous. This completes the proof.

We consider the following hypotheses in what follows.

$\left(A_{1}\right) \lim _{u \rightarrow 0^{+}} \sup _{t \in[0,1]} \frac{f(t, u)}{u}=0, \lim _{u \rightarrow 0^{+}} \sup _{t \in[0,1]} \frac{g(t, u)}{u}=0$;

$\left(A_{2}\right) \lim _{u \rightarrow+\infty} \inf _{t \in[0,1]} \frac{f(t, u)}{u}=+\infty, \lim _{u \rightarrow+\infty} \inf _{t \in[0,1]} \frac{g(t, u)}{u}=+\infty$;

$\left(A_{3}\right) \lim _{u \rightarrow 0^{+}} \inf _{t \in[0,1]} \frac{f(t, u)}{u}=+\infty, \lim _{u \rightarrow 0^{+}} \inf _{t \in[0,1]} \frac{g(t, u)}{u}=+\infty$;

$\left(A_{4}\right) \lim _{u \rightarrow+\infty} \sup _{t \in[0,1]} \frac{f(t, u)}{u}=0, \lim _{u \rightarrow+\infty} \sup _{t \in[0,1]} \frac{g(t, u)}{u}=0 ;$

$\left(A_{5}\right) f(t, u)$ and $g(t, u)$ are two increasing functions with respect to $u$, and there exists $N>0$ such that

$$
n_{1} f\left(t, \int_{0}^{1} n_{2} g(r, N) \mathrm{d} r\right)<N, \quad \text { for } t \in[0,1],
$$

where $n_{1}=\max _{0 \leq t, s \leq 1} G_{1}(t, s), n_{2}=\max _{0 \leq t, s \leq 1} G_{2}(t, s)$.

Theorem 3.1 Assume that hypotheses $\left(A_{1}\right)$ and $\left(A_{2}\right)$ hold. Then the boundary value problem (1.1) has at least one positive solution $(u, v)$.

Proof. By hypothesis $\left(A_{1}\right)$, we see that there exists $p_{1} \in(0,1)$ such that

$$
f(t, u) \leq \lambda_{1} u, \quad g(t, u) \leq \lambda_{2} u, \quad \text { for }(t, u) \in[0,1] \times\left(0, p_{1}\right),
$$

where $\lambda_{1}, \lambda_{2}>0$ and satisfy

$$
\frac{\lambda_{1}(\alpha-1)}{\Gamma(\alpha)} \int_{0}^{1} k_{1}(s) \mathrm{d} s \leq 1, \quad \frac{\lambda_{2} M_{0}}{\Gamma(\beta)} \int_{0}^{1} k_{2}(s) \mathrm{d} s \leq 1 .
$$


For $u \in P$ with $\|u\|=\frac{p_{1}}{2}$, we have

$$
\int_{0}^{1} G_{2}(s, r) g(r, u(r)) \mathrm{d} r \leq \int_{0}^{1} \frac{M_{0} k_{2}(r)}{\Gamma(\beta)} g(r, u(r)) \mathrm{d} r \leq \frac{\lambda_{2}\|u\|}{\Gamma(\beta)} \int_{0}^{1} M_{0} k_{2}(r) \mathrm{d} r \leq\|u\|=\frac{p_{1}}{2}<p_{1},
$$

then by (3.2) and (3.3), we get

$$
\begin{aligned}
\|T u\| & \leq \frac{\alpha-1}{\Gamma(\alpha)} \int_{0}^{1} k_{1}(s) f\left(s, \int_{0}^{1} G_{2}(s, r) g(r, u(r)) \mathrm{d} r\right) \mathrm{d} s \\
& \leq \frac{\lambda_{1}(\alpha-1)}{\Gamma(\alpha)} \int_{0}^{1} k_{1}(s) \int_{0}^{1} G_{2}(s, r) g(r, u(r)) \mathrm{d} r \mathrm{~d} s \\
& \leq \lambda_{1} \lambda_{2}\|u\| \frac{M_{0}(\alpha-1)}{\Gamma(\alpha) \Gamma(\beta)} \int_{0}^{1} k_{1}(s) \int_{0}^{1} k_{2}(r) \mathrm{d} r \mathrm{~d} s \\
& \leq\|u\| .
\end{aligned}
$$

Hence, if we choose $\Omega_{1}=\left\{u \in E:\|u\|<\frac{p_{1}}{2}\right\}$, then

$$
\|T u\| \leq\|u\|, \quad \text { for } u \in P \cap \partial \Omega_{1} .
$$

From hypothesis $\left(A_{2}\right)$, there exist positive constants $\mu_{1}, \mu_{2}, C_{1}$, and $C_{2}$ such that

$$
f(t, u) \geq \mu_{1} u-C_{1}, \quad g(t, u) \geq \mu_{2} u-C_{2}, \quad \text { for }(t, u) \in[0,1] \times[0,+\infty),
$$

where $\mu_{1}$ and $\mu_{2}$ satisfy

$$
\mu_{1} \int_{0}^{1} G_{1}(l, s) q_{2}(s) \mathrm{d} s \geq 1, \quad \frac{\mu_{2}(\beta-2)}{(\alpha-1) \Gamma(\beta)} \int_{0}^{1} q_{1}(r) k_{2}(r) \mathrm{d} r \geq 2,
$$

For $u \in P$ and $l \in(0,1)$, then by (3.5) and (3.6), we have

$$
\begin{aligned}
T u(l) & =\int_{0}^{1} G_{1}(l, s) f\left(s, \int_{0}^{1} G_{2}(s, r) g(r, u(r)) \mathrm{d} r\right) \mathrm{d} s \\
& \geq \int_{0}^{1} G_{1}(l, s)\left(\mu_{1} \int_{0}^{1} G_{2}(s, r) g(r, u(r)) \mathrm{d} r-C_{1}\right) \mathrm{d} s \\
& =\mu_{1} \int_{0}^{1} G_{1}(l, s) \int_{0}^{1} G_{2}(s, r) g(r, u(r)) \mathrm{d} r \mathrm{~d} s-C_{1} \int_{0}^{1} G_{1}(l, s) \mathrm{d} s \\
& \geq \mu_{1} \int_{0}^{1} G_{1}(l, s) \int_{0}^{1} G_{2}(s, r)\left(\mu_{2} u(r)-C_{2}\right) \mathrm{d} r \mathrm{~d} s-C_{1} \int_{0}^{1} G_{1}(l, s) \mathrm{d} s \\
& =\mu_{1} \mu_{2} \int_{0}^{1} G_{1}(l, s) \int_{0}^{1} G_{2}(s, r) u(r) \mathrm{d} r \mathrm{~d} s-C(l) \\
& \geq \mu_{1} \mu_{2} \frac{\beta-2}{(\alpha-1) \Gamma(\beta)}\|u\| \int_{0}^{1} G_{1}(l, s) q_{2}(s) \int_{0}^{1} q_{1}(r) k_{2}(r) \mathrm{d} r \mathrm{~d} s-C(l) \\
& \geq 2\|u\|-C(l), \quad
\end{aligned}
$$


where

$$
\begin{aligned}
C(l) & =\mu_{1} C_{2} \int_{0}^{1} G_{1}(l, s) \int_{0}^{1} G_{2}(s, r) \mathrm{d} r \mathrm{~d} s+C_{1} \int_{0}^{1} G_{1}(l, s) \mathrm{d} s \\
& \leq \frac{\mu_{1} C_{2} M_{0}}{\Gamma(\beta)} \int_{0}^{1} G_{1}(l, s) \int_{0}^{1} k_{2}(r) \mathrm{d} r \mathrm{~d} s+C_{1} \int_{0}^{1} G_{1}(l, s) \mathrm{d} s \\
& =C_{3},
\end{aligned}
$$

so,

$$
\mathrm{Tu}(l) \geq 2\|u\|-C_{3} .
$$

Thus, if we set $p_{2}>\max \left\{p_{1}, C_{3}\right\}$ and $\Omega_{2}=\left\{u \in E:\|u\|<p_{2}\right\}$, then

$$
\|T u\| \geq\|u\|, \quad \text { for } u \in P \cap \partial \Omega_{2} .
$$

Now, from (3.4), (3.7), and Lemma 2.7, we guarantee that $T$ has a fix point $u \in P \cap\left(\bar{\Omega}_{2} \backslash \Omega_{1}\right)$, and clearly $(u, v)$ is a positive solution of (1.1). The proof is completed.

Theorem 3.2 Assume that hypotheses $\left(A_{3}\right)$ and $\left(A_{4}\right)$ hold. Then the boundary value problem (1.1) has at least one positive solution $(u, v)$.

Proof. By hypothesis $\left(A_{3}\right)$, we see that there exists $p \in(0,1)$ such that

$$
f(t, u) \geq \eta_{1} u, \quad g(t, u) \geq \eta_{2} u, \quad \text { for }(t, u) \in[0,1] \times(0, p),
$$

where $\eta_{1}, s_{2}>0$ and satisfy

$$
\eta_{1} \int_{0}^{1} G_{1}(l, s) q_{2}(s) \mathrm{d} s \geq 1, \quad \frac{\eta_{2}(\beta-2)}{(\alpha-1) \Gamma(\beta)} \int_{0}^{1} q_{1}(r) k_{2}(r) \mathrm{d} r \geq 1,
$$

From $g(t, 0) \equiv 0$ and the continuity of $g$, then there exists $p_{3} \in(0,1)$ such that

$$
g(t, u) \leq \frac{p}{M_{0} \int_{0}^{1} k_{2}(r) \mathrm{d} r}, \quad \text { for }(t, u) \in[0,1] \times\left(0, p_{3}\right) .
$$

For $u \in P$ with $\|u\|=p_{3}$, we have

$$
\left.\int_{0}^{1} G_{2}(s, r) g(r, u)(r)\right) \mathrm{d} r \leq \int_{0}^{1} G_{2}(s, r) \frac{p}{M_{0} \int_{0}^{1} k_{2}(r) \mathrm{d} r} \mathrm{~d} r<p,
$$

for $l \in(0,1)$, by (3.8) and (3.9), we get

$$
\begin{aligned}
T u(l) & =\int_{0}^{1} G_{1}(l, s) f\left(s_{1} \int_{0}^{1} G_{2}(s, r) g(r, u(r)) \mathrm{d} r\right) \mathrm{d} s \\
& \geq \eta_{1} \int_{0}^{1} G_{1}(l, s) \int_{0}^{1} G_{2}(s, r) g(r, u(r)) \mathrm{d} r \mathrm{~d} s \\
& \geq \eta_{1} \eta_{2} \int_{0}^{1} G_{1}(l, s) \int_{0}^{1} G_{2}(s, r) u(r) \mathrm{d} r \mathrm{~d} s \\
& \geq \eta_{1} \eta_{2} \frac{\beta-2}{(\alpha-1) \Gamma(\beta)}\|u\| \int_{0}^{1} G_{1}(l, s) q_{2}(s) \int_{0}^{1} g_{1}(r) k_{2}(r) \mathrm{d} r \mathrm{~d} s \\
& \geq\|u\|,
\end{aligned}
$$


Hence, if we choose $\Omega_{3}=\left\{u \in E:\|u\|<p_{3}\right\}$, then

$$
\|T u\| \leq\|u\|, \quad \text { for } u \in P \cap \partial \Omega_{3} .
$$

From hypothesis $\left(A_{4}\right)$, there exist positive constants $\delta_{1}, \delta_{2}, C_{4}$, and $C_{5}$ such that

$$
f(t, u) \geq \delta_{1} u+C_{4}, \quad g(t, u) \geq \delta_{2} u+C_{5}, \quad \text { for }(t, u) \in[0,1] \times[0,+\infty),
$$

where $\delta_{1}$ and $\delta_{2}$ satisfy

$$
\frac{\delta_{1}(\alpha-1)}{\Gamma(\alpha)} \int_{0}^{1} k_{1}(s) \mathrm{d} s \leq \frac{1}{2}, \quad \frac{\delta_{2} M_{0}}{\Gamma(\beta)} \int_{0}^{1} k_{2}(r) \mathrm{d} r \leq \frac{1}{2} .
$$

Then by (3.11) and (3.12), we have

$$
\begin{aligned}
\|T u\| & \leq \frac{\alpha-1}{\Gamma(\alpha)}=\int_{0}^{1} k_{1}(s) f\left(s_{1} \int_{0}^{1} G_{2}(s, r) g(r, u(r)) \mathrm{d} r\right) \mathrm{d} s \\
& \leq \frac{\alpha-1}{\Gamma(\alpha)} \int_{0}^{1} k_{1}(s)\left(\delta_{1} \int_{0}^{1} G_{2}(s, r) g(r, u(r)) \mathrm{d} r+C_{4}\right) \mathrm{d} s \\
& =\frac{\delta_{1}(\alpha-1)}{\Gamma(\alpha)} \int_{0}^{1} k_{1}(s) \int_{0}^{1} G_{2}(s, r) g(r, u(r)) \mathrm{d} r \mathrm{~d} s+\frac{C_{4}(\alpha-1)}{\Gamma(\alpha)} \int_{0}^{1} k_{1}(s) \mathrm{d} s \\
& \leq \frac{\delta_{1}(\alpha-1)}{\Gamma(\alpha)} \int_{0}^{1} k_{1}(s) \int_{0}^{1} \frac{M_{0} k_{2}(r)}{\Gamma(\beta)}\left(\delta_{2} u(r)+C_{5}\right) \mathrm{d} r \mathrm{~d} s+\frac{C_{4}(\alpha-1)}{\Gamma(\alpha)} \int_{0}^{1} k_{1}(s) \mathrm{d} s \\
& \leq \frac{\delta_{1} \delta_{2} M_{0}(\alpha-1)}{\Gamma(\alpha) \Gamma(\beta)}\|u\| \int_{0}^{1} k_{1}(s) \int_{0}^{1} k_{2}(r) \mathrm{d} r \mathrm{~d} s-C_{6} \\
& \leq \frac{1}{4}\|u\|+C_{6},
\end{aligned}
$$

where

$$
C_{6}=\frac{\delta_{1} C_{5} M_{0}(\alpha-1)}{\Gamma(\alpha) \Gamma(\beta)} \int_{0}^{1} k_{1}(s) \int_{0}^{1} k_{2}(r) \mathrm{d} r \mathrm{~d} s+\frac{C_{4}(\alpha-1)}{\Gamma(\alpha)} \int_{0}^{1} k_{1}(s) \mathrm{d} s .
$$

Thus, if we set $p_{4}>\max \left\{2 p_{3}, 2 C_{6}\right\}$ and $\Omega_{4}=\left\{u \in E:\|u\|<p_{4}\right\}$, then

$$
\|T u\| \leq\|u\|, \quad \text { for } u \in P \cap \partial \Omega_{4} .
$$

Now, from (3.10), (3.13), and Lemma 2.7, we guarantee that $T$ has a fix point $u \in P \cap\left(\bar{\Omega}_{2} \backslash \Omega_{1}\right)$, and clearly $(u, v)$ is a positive solution of (1.1). The proof is completed.

Theorem 3.3 Assume that hypotheses $\left(A_{2}\right),\left(A_{3}\right)$, and $\left(A_{5}\right)$ hold. Then the boundary value problem (1.1) has at least two positive solutions $\left(u_{1}, v_{1}\right)$ and $\left(u_{2}, v_{2}\right)$. 
Proof. Set $B_{N}=\{u \in E:\|u\|<N\}$. From $\left(A_{5}\right)$, for $u \in P \cap \partial B_{N}$, then we have

$$
\begin{aligned}
\|T u\| & =\max _{0 \leq t \leq 1}|T u(t)| \\
& =\max _{0 \leq t \leq 1}\left|\int_{0}^{1} G_{1}(t, s) f\left(s, \int_{0}^{1} G_{2}(s, r) g(r, u(r)) \mathrm{d} r\right) \mathrm{d} s\right| \\
& \leq n_{1} \int_{0}^{1} f\left(s, \int_{0}^{1} G_{2}(s, r) g(r, u(r)) \mathrm{d} r\right) \mathrm{d} s \\
& \leq n_{1} \int_{0}^{1} f\left(s_{,} \int_{0}^{1} n_{2} g(r, u(r)) \mathrm{d} r\right) \mathrm{d} s \\
& \left.<n_{1} \int_{0}^{1} f\left(s_{,} \int_{0}^{1} n_{2} g(r, N)\right) \mathrm{d} r\right) \mathrm{d} s<N .
\end{aligned}
$$

Thus, $\|T u\|<\|u\|, \forall u \in P \cap \partial B_{N}$. By $\left(A_{2}\right)$ and $\left(A_{3}\right)$, we can get

$$
\begin{aligned}
& \|T u\| \geq\|u\|, \quad \forall u \in P \cap \partial \Omega_{2}, \\
& \|T u\| \geq\|u\|, \quad \forall u \in P \cap \partial \Omega_{3} .
\end{aligned}
$$

So, we can choose $p_{2}, p_{3}$, and $N$ such that $p_{3}<N<p_{2}$ and satisfy the above three inequalities. By Lemma 2.8, we guarantee that $T$ has two fix points $u_{1} \in P \cap\left(\bar{\Omega}_{2} \backslash B_{N}\right)$ and $u_{2} \in P \cap\left(\overline{B_{N}} \backslash \Omega_{3}\right)$. Then the boundary value problem (1.1) at least two positive solutions $\left(u_{1}, v_{1}\right)$ and $\left(u_{2}, v_{2}\right)$. This completes the proof.

In fact, from (3.1), we can also obtain the following integral equation

$$
v(t)=\int_{0}^{1} G_{2}(t, s) g\left(s, \int_{0}^{1} G_{1}(s, r) f(r, v(r)) \mathrm{d} r\right) \mathrm{d} s, \quad t \in[0,1] .
$$

Define the cone $P^{\prime} \subset E$ by

$$
P^{\prime}=\left\{v \in E: v(t) \geq \frac{(\alpha-2) q_{2}(t)}{M_{0}}\|v\|, t \in[0,1]\right\} .
$$

We define an operator $T^{\prime}: P^{\prime} \rightarrow E$ as follows

$$
T^{\prime} v(t)=\int_{0}^{1} G_{2}(t, s) g\left(s, \int_{0}^{1} G_{1}(s, r) f(r, v(r)) \mathrm{d} r\right) \mathrm{d} s, \quad t \in[0,1] .
$$

For $v \in P^{\prime}, 0 \leq t \leq 1$, by Lemma 2.6,

$$
\begin{aligned}
\left\|T^{\prime} v\right\| & =\max _{0 \leq t \leq 1}\left|T^{\prime} v(t)\right| \\
& =\max _{0 \leq t \leq 1}\left|\int_{0}^{1} G_{2}(t, s) g\left(s, \int_{0}^{1} G_{1}(s, r) f(r, v(r)) \mathrm{d} r\right) \mathrm{d} s\right| \\
& \leq \frac{1}{\Gamma(\alpha)} \int_{0}^{1} M_{0} k_{2}(s) g\left(s, \int_{0}^{1} G_{1}(s, r) f(r, v(r)) \mathrm{d} r\right) \mathrm{d} s,
\end{aligned}
$$




$$
\begin{aligned}
T^{\prime} v(t) & =\int_{0}^{1} G_{2}(t, s) g\left(s, \int_{0}^{1} G_{1}(s, r) f(r, v(r)) \mathrm{d} r\right) \mathrm{d} s \\
& \geq \int_{0}^{1} \frac{(\alpha-2)}{\Gamma(\alpha)} q_{2}(t) k_{2}(s) g\left(s, \int_{0}^{1} G_{1}(s, r) f(r, v(r)) \mathrm{d} r\right) \mathrm{d} s \\
& \geq \frac{(\alpha-2) q_{2}(t)}{M_{0}}\|T v\| .
\end{aligned}
$$

Thus we have $T^{\prime}\left(P^{\prime}\right) \subset P^{\prime}$.

The operator $T: P^{\prime} \rightarrow P^{\prime}$ is continuous in view of continuity of $G(t, s), f(t, u)$, and $g(t$, $u)$. For any bounded set $M^{\prime}, T^{\prime}\left(M^{\prime}\right)$ is uniformly bounded and equicontinuous. This proof is similar to that of Lemma 3.1 in [21], so is omitted. By means of Arzela-Ascoli Theorem, $T: P^{\prime} \rightarrow P^{\prime}$ is completely continuous.

Remark 3.1 Theorems 3.1 and 3.2 also hold for the boundary value problem (1.1). Proof. This proof is similar to that of Theorems 3.1 and 3.2, so is omitted.

Theorem 3.4 If conditions $\left(A_{5}\right)$ in the Theorem 3.3 is replaced by

$\left(A_{5}^{\prime}\right) f(t, u)$ and $g(t, u)$ are two increasing functions with respect to $u$, and there exists $N>0$ such that

$$
n_{2} g\left(t, \int_{0}^{1} n_{1} f\left(r, N^{\prime}\right) d r\right)<N^{\prime}, \quad \text { for } t \in[0,1],
$$

where $n_{1}=\max _{0} \leq t, s \leq 1 G_{1}(t, s), n_{2}=\max _{0 \leq t, s \leq 1} G_{2}(t, s)$.

Then the conclusion of Theorem 3.3 also holds.

Proof. This proof is similar to that of Theorem 3.3, so is omitted.

Remark 3.2 In this article, conditions $f(t, 0) \equiv 0$ and $g(t, 0) \equiv 0$ are too strong for the boundary value problem (1.1). So, we will give some new existence criteria for the boundary value problem (1.1) without conditions $f(t, 0) \equiv 0$ and $g(t, 0) \equiv 0$ in a new paper.

\section{Examples}

In this section, we will present examples to illustrate the main results.

Example 4.1 Consider the system of nonlinear differential equations

$$
\left\{\begin{array}{l}
-\frac{5}{2} \\
-D_{0^{+}} u(t)=v(v+t-1), 0<t<1 \\
\frac{{ }^{2}}{2} u(t)=u(u+t-1), \quad 0<t<1 \\
u(0)=u(1)=u^{\prime}(0)=v(0)=v(1)=v^{\prime}(0)=v^{\prime}(1)=0 .
\end{array}\right.
$$

Choose $f(t, v)=v(v+t-1), g(t, u)=u(u+t-1)$. Then

$$
\begin{aligned}
& \lim _{u \rightarrow 0^{+}} \sup _{t \in[0,1]} \frac{f(t, u)}{u}=\lim _{u \rightarrow 0^{+}} \sup _{t \in[0,1]} \frac{g(t, u)}{u}=\lim _{u \rightarrow 0^{+}} \sup _{t \in[0,1]}(u+t-1)=0, \\
& \lim _{u \rightarrow+\infty} \inf _{t \in[0,1]} \frac{f(t, u)}{u}=\lim _{u \rightarrow+\infty} \inf _{t \in[0,1]} \frac{g(t, u)}{u}=\lim _{u \rightarrow+\infty} \inf _{t \in[0,1]}(u+t-1)=+\infty .
\end{aligned}
$$


So $\left(A_{1}\right)$ and $\left(A_{2}\right)$ hold. Thus, by Theorem 3.1, the boundary value problem (4.1) has a positive solution.

Example 4.2 Discuss the system of nonlinear differential equations

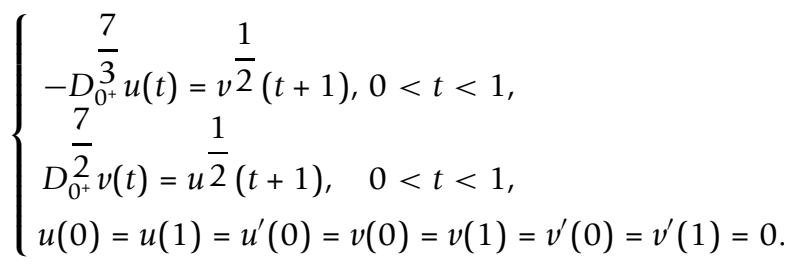

$$
\begin{aligned}
& \text { Choose } f(t, v)=v^{\frac{1}{2}}(t+1)^{\prime} g(t, u)=u^{\frac{1}{2}}(t+1)^{\text {Then }} \\
& \lim _{u \rightarrow 0^{+}} \inf _{t \in[0,1]} \frac{f(t, u)}{u}=\lim _{u \rightarrow 0^{+}} \inf _{t \in[0,1]} \frac{g(t, u)}{u}=\lim _{u \rightarrow 0^{+}} \inf _{t \in[0,1]} \frac{t+1}{u^{\frac{1}{2}}}=+\infty, \\
& \lim _{u \rightarrow+\infty} \sup _{t \in[0,1]} \frac{f(t, u)}{u}=\lim _{u \rightarrow+\infty} \sup _{t \in[0,1]} \frac{g(t, u)}{u}=\lim _{u \rightarrow+\infty} \sup _{t \in[0,1]} \frac{t+1}{u^{\frac{1}{2}}}=0 .
\end{aligned}
$$

So $\left(A_{3}\right)$ and $\left(A_{4}\right)$ hold. Thus, by Theorem 3.2, the boundary value problem (4.2) has a positive solution.

\section{Acknowledgements}

This research is supported by the Natural Science Foundation of China (11071143, 60904024, 11026112), China Postdoctoral Science Foundation funded project (200902564), and supported by Shandong Provincial Natural Science Foundation (ZR2010AL002, ZR2009AL003, Y2008A28), also supported by University of Jinan Research Funds for Doctors (XBS0843) and University of Jinan Innovation Funds for Graduate Students (YCX09014).

\section{Author details}

${ }^{1}$ School of Science, University of Jinan, Jinan 250022, Shandong, PR China ${ }^{2}$ Department of Mathematics and Statistics, Missouri University of Science and Technology, Rolla, MI 65409-0020, USA ${ }^{3}$ School of Control Science and Engineering, Shandong University, Jinan 250061, Shandong, PR China

\section{Authors' contributions}

The work presented here was carried out in collaboration between all authors.

$Y Z$ carried out the design of the study, the statistical analysis and drafted the manuscript.

SS and ZH conceived, instructed the design of the study and polished the manuscript.

WF participated discussion. All authors read and approved the final manuscript.

\section{Competing interests}

The authors declare that they have no competing interests.

Received: 11 November 2010 Accepted: 14 June 2011 Published: 14 June 2011

\section{References}

1. Miller KS, Ross B: An Introduction to the Fractional Calculus and Fractional Differential Equation. Wiley, New York; 1993.

2. Oldham KB, Spanier J: The Fractional Calculus. Academic Press, New York; 1974.

3. Podlubny I: Fractional Differential Equations, Mathematics in Science and Engineering. Academic Press, New York, Lindon, Toronto; 1999.

4. Samko SG, Kilbas AA, Marichev Ol: Fractional Integral and Derivative. Theory and Applications Gordon and Breach, Yverdon; 1993

5. Kilbas AA, Trujillo JJ: Differential equations of fractional order: methods, results and problem. / App/ Anal 2001, 78:153-192.

6. Kilbas AA, Trujillo JJ: Differential equations of fractional order: methods, results and problems. // Appl Anal 2002, 81:435-493.

7. Delbosco D, Rodino L: Existence and uniqueness for a nonlinear fractional differential equation. J Math Anal Appl 1996, 204:609-625.

8. Li Q, Sun S: On the existence of positive solutions for initial value problem to a class of fractional differential equation. Proceedings of the 7th Conference on Biological Dynamic System and Stability of Differential Equation World Academic Press Chongqing, II; 2010, 886-889.

9. Li Q, Sun S, Zhang M, Zhao Y: On the existence and uniqueness of solutions for initial value problem of fractional differential equations. J Univ Jinan 2010, 24:312-315. 
10. Li Q, Sun $S$, Han Z, Zhao Y: On the existence and uniqueness of solutions for initial value problem of nonlinear fractional differential equations. 2010 Sixth IEEE/ASME International Conference on Mechatronic and Embedded Systems and Applications, Qingdao 2010, 452-457.

11. Zhang S: The existence of a positive solution for nonlinear fractional differential equation. J Math Anal Appl 2000, 252:804-812.

12. Jafari $\mathrm{H}$, Gejji VD: Positive solutions of nonlinear fractional boundary value problems using Adomian decomposition method. Appl Math Comput 2006, 180:700-706.

13. Bai Z, Lü H: Positive solutions for boundary value problem of nonlinear fractional differential equation. J Math Anal Appl 2005, 311:495-505.

14. Qiu T, Bai Z: Existence of positive solutions for singular fractional equations. Electron J Differ Equ 2008, 146:1-9.

15. Zhang S: Positive solutions for boundary-value problems of nonlinear fractional differential equations. Electron $J$ Differ Equ 2006, 36:1-12.

16. Zhang $M$, Sun $S$, Zhao $Y$, Yang D: Existence of positive solutions for boundary value problems of fractional differential equations. J Univ Jinan 2010, 24:205-208.

17. Zhao Y, Sun S: On the existence of positive solutions for boundary value problems of non-linear fractional differential equations. Proceedings of the 7th Conference on Biological Dynamic System and Stability of Differential Equation World Academic Press Chongqing, II; 2010, 682-685.

18. Zhao $Y$, Sun $S$, Han Z, Zhang M: Existence on positive solutions for boundary value problems of singular nonlinear fractional differential equations. 2010 Sixth IEEE/ASME International Conference on Mechatronic and Embedded Systems and Applications, Qingdao 2010, 480-485.

19. Zhao Y, Sun S, Han Z, Li Q: The existence of multiple positive solutions for boundary value problems of nonlinear fractional differential equations. Commun Nonlinear Sci Numer Simul 2011, 16:2086-2097.

20. Yu Y, Jiang D: Multiple Positive Solutions for the Boundary Value Problem of A Nonlinear Fractional Differential Equation. Northeast Normal University; 2009.

21. Xu X, Jiang D, Yuan C: Multiple positive solutions for the boundary value problem of a nonlinear fractional differential equation. Nonlinear Anal 2009, 71:4676-4688.

22. Ladaci S, Loiseau JL, Charef A: Fractional order adaptive high-gain controllers for a class of linear systems. Commun Nonlinear Sci Numer Simul 2008, 13:707-714.

23. Yang A, Ge W: Positive solutions for boundary value problems of N-Dimension nonlinear fractional differential system. Bound Value Probl 2008, 2008:1-15.

24. Bai C, Fang J: The existence of a positive solution for a singular coupled system of nonlinear fractional differential equations. Appl Math Comput 2004, 150:611-621.

25. Su X: Boundary value problem for a coupled system of nonlinear fractional differential equations. Appl Math Lett 2009, 22:64-69.

26. Geji VD: Positive solutions of a system of nonlinear fractional differential equations. J Math Anal Appl 2005, 302:56-64.

27. Wang J, Xiang H, Liu Z: Positive solution to nonzero boundary values problem for a coupled system of nonlinear fractional differential equations. Int J Differ Equ 2010, 2010:1-12.

28. Ahmad B, Nieto JJ: Existence results for a coupled system of nonlinear fractional differential equations with threepoint boundary conditions. Comput Math Appl 2009, 58:1838-1843.

29. Ahmad B, Alsaedi A: Existence and uniqueness of solutions for coupled systems of higher-order nonlinear fractional differential equations. Fixed Point Theory Appl 2010, 2010:1-17.

30. Babakhani A: Positive solutions for system of nonlinear fractional differential equations in two dimensions with delay. Abstr Appl Anal 2010, 2010:1-16.

31. Kilbas AA, Srivastava HH, Trujillo JJ: Theory and Applications of Fractional Differential Equations. Elsevier, Amsterdam; 2006.

32. Krasnoselskii MA: Positive Solution of Operator Equation. Noordho, Groningen 1964.

33. Kang P, Wei Z: Three positive solutions of singular nonlocal boundary value problems for systems of nonlinear second-order ordinary differential equations. Nonlinear Anal 2009, 70:444-451.

doi:10.1186/1687-1847-2011-10

Cite this article as: Zhao et al:: Positive solutions for a coupled system of nonlinear differential equations of

mixed fractional orders. Advances in Difference Equations 2011 2011:10.

\section{Submit your manuscript to a SpringerOpen ${ }^{\circ}$ journal and benefit from:}

- Convenient online submission

- Rigorous peer review

- Immediate publication on acceptance

- Open access: articles freely available online

- High visibility within the field

- Retaining the copyright to your article

Submit your next manuscript at $\boldsymbol{s p r i n g e r o p e n . c o m ~}$ 